\title{
NOVEL OPTIMAL RECURSIVE FILTER FOR STATE AND FAULT ESTIMATION OF LINEAR STOCHASTIC SYSTEMS WITH UNKNOWN DISTURBANCES
}

\author{
KARIM KHÉMIRI*, FAYÇAL BEN HMIDA* , José RAGOT **, MONCEF GOSSA* \\ ${ }^{*}$ Research Unit on Control, Monitoring and Safety of Systems (C3S) \\ High School of Sciences and Techniques of Tunis (ESSTT), 5 av. Taha Hussein, BP 56-1008 Tunis, Tunisia \\ e-mail:kemiri_karim@yahoo.fr, \{faycal.benhmida, moncef.gossa\}@esstt.rnu.tn \\ ${ }^{* *}$ Research Centre in Automation of Nancy (CRAN) \\ Nancy University, CNRS, BP 239, 54506 Vandoeuvre Cedex, France \\ e-mail: Jose.Ragot@ensem.inpl-nancy. fr
}

\begin{abstract}
This paper studies recursive optimal filtering as well as robust fault and state estimation for linear stochastic systems with unknown disturbances. It proposes a new recursive optimal filter structure with transformation of the original system. This transformation is based on the singular value decomposition of the direct feedthrough matrix distribution of the fault which is assumed to be of arbitrary rank. The resulting filter is optimal in the sense of the unbiased minimum-variance criteria. Two numerical examples are given in order to illustrate the proposed method, in particular to solve the estimation of the simultaneous actuator and sensor fault problem and to make a comparison with the existing literature results.
\end{abstract}

Keywords: Kalman filtering, minimum-variance estimation, state estimation, fault estimation, unknown disturbances, linear discrete-time systems.

\section{Introduction}

Joint fault and state estimation of linear time-varying stochastic systems with unknown disturbances is considered. This problem is solved by using an unknown input filtering approach to produce unknown disturbance decoupled joint fault-state estimation. This may be achieved by making use of unbiased minimum-variance estimation. The proposed filter can play a significant role in several applications, e.g., the model-based Fault Detection and Isolation (FDI) problem (Ben Hmida et al., 2010; Chen and Patton, 1996; 1999) and the Fault Tolerant Control (FTC) problem (Blanke et al., 2006).

The subject of Unknown Input Filtering (UIF) has been studied extensively in the past three decades. A first approach was developed by Kitanidis (1987) using an Unbiased Minimum-Variance (UMV) estimation technique. This approach assumes that no prior knowledge about the dynamical evolution of the unknown inputs is available. Since then, many authors have extended Kitanidis's filter, (e.g., Cheng et al., 2009; Gillijns and Moor, 2007b).

A parameterizing technique is used to obtain an optimal filter under less restrictive conditions as in the work of Darouach and Zasadzinski (1997). Their results are only limited to treat the state estimation problem. However, Hsieh (2000) as well as Gillijns and Moor (2007a) investigated the joint input and state estimation problem using an innovation filtering technique. All the previous works are, nevertheless, limited to linear systems without direct feedthrough of the unknown input to the output.

In the case where the unknown inputs or disturbances affect both the system state and the output, the state estimation problem was addressed by Hou and Patton (1998), Darouach et al. (2003) as well as Cheng et al. (2009). Hou and Patton (1998) developed a straightforward state estimation method to design an optimal filter. A parameterizing technique was used by Darouach et al. (2003) to derive an Optimal Estimator Filter (OEF). Recently, Cheng et al. (2009) developed a recursive optimal filter with global optimality in the sense of unbiased minimum-variance. In addition, they established a necessary and sufficient condition for the convergence and stability of this filter. However, all these results are solely limited to the estimation of the state, not the input.

The joint input and state estimation problem for linear systems with direct feedthrough was treated by Gillijns and Moor (2007b) as well as Hsieh (2009). In the for- 
mer work the Recursive Three Step Filter (RTSF) is derived where the input estimation is based on the Weighted Least-Squares (WLS) approach and the state estimation problem is solved by Kitanidis's method. The RTSF is usable if only the direct feedthrough matrix has full rank. Recently, the case of arbitrary rank was proposed by Hsieh (2009). The designed optimal filter, known as the ERTSF (Extended RTSF), made it possible to estimate an UMV state and input. But, in certain cases, the filter suffers from a degradation of the input estimates.

The FDI problem for linear systems without and with unknown disturbances has been thoroughly investigated (Nikoukhah, 1994; Keller, 1998; 1999; Keller and Sauter 2011; Jamouli et al., 2003, Chen and Patton, 1996; 1999; Ben Hmida et al., 2010). Keller (1999) designed a fullorder Kalman filter under a particular eigenstructure assignment. A new state filtering strategy was developed to detect and isolate multiple faults appearing simultaneously or sequentially. Keller and Sauter (2011) presented a restricted diagonal detection filter for multiple fault detection and isolation. The proposed filter makes it possible to generate a minimum variance white innovation sequence having directional properties in response to each fault. For detection and isolation of multiple impulsive faults, a bank of Generalised Likelihood Ratio (GLR) tests is applied to the innovation sequence generated by the detection filter.

Unfortunately, these works do not attack the case where unknown disturbances may affect the systems and produce a false alarm. To deal with this case for systems with unknown disturbances, we can, according to Nikoukhah (1994), develop a robust fault detection and isolation in continuous time by using the error innovation technique to generate unbiased white residual signals. The fault is diagnosed by a statistical testing of the whiteness, mean and covariance of residuals.

A new method was developed by Keller (1998) to detect and isolate multiple faults appearing simultaneously or sequentially in Linear Time-Invariant (LTI) stochastic discrete-time systems with unknown inputs. That method consists of generating directional residuals using an isolation filter. Jamouli et al. (2003) presented a Fault Isolation Filter (FIF) for fault detection in linear stochastic systems affected by disturbances. The filter will generate a directional residual decoupled from the unknown input allowing the treatment of multiple faults appearing simultaneously or sequentially.

Chen and Patton $(1996 ; 1999)$ studied the optimal filtering and robust fault diagnosis problem for stochastic systems with unknown disturbances. An optimal observer was proposed, which can produce disturbance decoupled state estimation with minimum-variance for linear time-varying systems with both noise and unknown disturbances. The output estimation error with disturbance decoupling is used as a residual signal. After that, a statistical testing procedure is applied to examine the residual and to diagnose faults. Nevertheless, the simultaneous actuator and sensor faults problem has not been studied.

Recently, Ben Hmida et al. (2010) presented a new recursive filter to joint fault and state estimation of linear time-varying discrete-time systems in the presence of unknown disturbances. The method is based on the assumption that no prior knowledge about the dynamical evolution of the fault and the unknown disturbances is available. The filter has two advantages: it considers an arbitrary direct feedthrough matrix of the fault and allows for estimation of multiple faults. However, in certain cases the obtained filter may suffer from poor fault estimation quality.

In this paper, we extend the version of Ben Hmida et al. (2010) to further propose a new recursive optimal filter structure. The fault affects both the state and output equations where the direct feedthrough matrix has arbitrary rank, whereas the unknown disturbances only affect the state system equation, without any prior information about their dynamical evolution. This filter has been obtained through a linear transformation of the original system. Next, we use a linear Unbiased Minimum-Variance (UMV) estimation. The estimation of the fault is obtained by using a weighted least-squares approach. Thus, the resulting filter will be applied to solve a simultaneous actuator and sensor fault estimation.

The remainder of this paper is organized as follows. Section 2 states the problem of interest. In Section 3 we design the proposed filter. Thus Section 4 presents an illustrative example.

\section{Problem statement}

Consider a linear stochastic discrete-time system in the following form:

$$
\begin{aligned}
x_{k+1} & =A_{k} x_{k}+B_{k} u_{k}+F_{k}^{x} f_{k}+E_{k}^{x} d_{k}+w_{k}, \\
y_{k} & =H_{k} x_{k}+F_{k}^{y} f_{k}+v_{k},
\end{aligned}
$$

where $x_{k} \in \mathbb{R}^{n}$ is the state vector, $y_{k} \in \mathbb{R}^{m}$ is the observation vector, $u_{k} \in \mathbb{R}^{r}$ is the known input vector, $f_{k} \in \mathbb{R}^{p}$ is the additive fault vector and $d_{k} \in \mathbb{R}^{q}$ is the unknown disturbances vector. Here $w_{k}$ and $v_{k}$ are uncorrelated white noise sequences with zero means, and the covariances matrices $Q_{k}=\mathcal{E}\left[w_{k} w_{k}^{T}\right] \geq 0$ and $R_{k}=\mathcal{E}\left[v_{k} v_{k}^{T}\right]>0$, respectively. The matrices $A_{k}, H_{k}, F_{k}^{x}, E_{k}^{x}$ and $F_{k}^{y}$ are known and have appropriate dimensions. We assume that $\left(A_{k}, H_{k}\right)$ is observable, $m \geq p+q$ and the initial state is uncorrelated with the white noise processes $w_{k}$ and $v_{k}$. The initial state $x_{0}$ is a Gaussian random variable with $\mathcal{E}\left[x_{0}\right]=\hat{x}_{0}$ and $\mathcal{E}\left[\left(x_{0}-\hat{x}_{0}\right)\left(x_{0}-\hat{x}_{0}\right)^{T}\right]=P_{0}^{x}$, where $\mathcal{E}[\cdot]$ denotes the expectation operator. 
The aim of this paper is to extend the results of Ben Hmida et al. (2010) in order to derive a new recursive optimal filter structure to obtain a better fault and state estimation when $0<\operatorname{rank}\left(F_{k}^{y}\right) \leq p$ in spite of the presence of the unknown disturbances $d_{k}$.

Initially, we seek to change the coordinates of the system (1) and (2) by using the technique developed by Cheng et al. (2009). Let $r_{k}=\operatorname{rank}\left(F_{k}^{y}\right)<p$. Then the singular value decomposition of the matrix $F_{k}^{y}$ is given by

$$
F_{k}^{y}=\left[\begin{array}{ll}
U_{1, k} & U_{2, k}
\end{array}\right]\left[\begin{array}{cc}
\Sigma_{k} & 0 \\
0 & 0
\end{array}\right]\left[\begin{array}{c}
V_{1, k}^{T} \\
V_{2, k}^{T}
\end{array}\right],
$$

where we have $\Sigma_{k} \in \mathbb{R}^{r_{k} \times r_{k}}, U_{1, k} \in \mathbb{R}^{m \times r_{k}}, U_{2, k} \in$ $\mathbb{R}^{m \times\left(m-r_{k}\right)}, V_{1, k} \in \mathbb{R}^{p \times r_{k}}$ and $V_{2, k} \in \mathbb{R}^{p \times\left(p-r_{k}\right)}$. $\left[\begin{array}{ll}U_{1, k} & U_{2, k}\end{array}\right]$ and $\left[\begin{array}{lll}V_{1, k} & V_{2, k}\end{array}\right]$ are unitary matrices.

There exists a transformation matrix of the from $T_{k}=\left[\begin{array}{ll}T_{1, k}^{T} & T_{2, k}^{T}\end{array}\right]^{T}$ such that the system (1) and (2) is written as (Cheng et al., 2009)

$$
\begin{aligned}
x_{k+1}= & A_{k} x_{k}+B_{k} u_{k}+F_{1, k}^{x} f_{1, k}+F_{2, k}^{x} f_{2, k} \\
& +E_{k}^{x} d_{k}+w_{k}, \\
z_{1, k}= & H_{1, k} x_{k}+\Sigma_{k} f_{1, k}+v_{1, k}, \\
z_{2, k}= & H_{2, k} x_{k}+v_{2, k},
\end{aligned}
$$

where

$$
\begin{aligned}
v_{1, k} & =T_{1, k} v_{k}, & v_{2, k} & =T_{2, k} v_{k}, \\
z_{1, k} & =T_{1, k} y_{k} \in \mathbb{R}^{r_{k}}, & z_{2, k} & =T_{2, k} y_{k} \in \mathbb{R}^{\left(m-r_{k}\right)}, \\
F_{1, k}^{x} & =F_{k}^{x} V_{1, k}, & F_{2, k}^{x} & =F_{k}^{x} V_{2, k}, \\
H_{1, k} & =T_{1, k} H_{k}, & H_{2, k} & =T_{2, k} H_{k},
\end{aligned}
$$

with the assumption that

$$
\begin{aligned}
\operatorname{rank}\left(H_{2, k} F_{2, k-1}^{x}\right) & =\operatorname{rank}\left(F_{2, k-1}^{x}\right), \\
\operatorname{rank}\left(H_{2, k} E_{k-1}^{x}\right) & =\operatorname{rank}\left(E_{k-1}^{x}\right) .
\end{aligned}
$$

Since $\left[V_{1, k} V_{2, k}\right]$ is a unitary matrix, it follows that the fault must be reconstructed through its two components $f_{1, k}$ and $f_{2, k}$ according to

$$
f_{k}=V_{1, k} f_{1, k}+V_{2, k} f_{2, k}
$$

The transformation matrix $T_{k}$ is given by

$$
\begin{aligned}
T_{k}= & {\left[\begin{array}{cc}
I_{r_{k}} & -U_{1, k}^{T} R_{k} U_{2, k}\left(U_{2, k}^{T} R_{k} U_{2, k}\right)^{-1} \\
0 & I_{\left(m-r_{k}\right)}
\end{array}\right] } \\
& \times\left[\begin{array}{c}
U_{1, k}^{T} \\
U_{2, k}^{T}
\end{array}\right] .
\end{aligned}
$$

Cheng et al. (2009) define $R_{1, k}$ and $R_{2, k}$ as the variance of $v_{1, k}$ and $v_{2, k}$, respectively, and $R_{12}(k, i)$ as their covariance. Then it follows that

$$
\begin{aligned}
R_{1, k}= & \mathcal{E}\left[v_{1, k} v_{1, k}^{T}\right], \\
= & U_{1, k}^{T} R_{k} U_{1, k}-U_{1, k}^{T} R_{k} U_{2, k} \\
& \times\left(U_{2, k}^{T} R_{k} U_{2, k}\right)^{-1} U_{2, k}^{T} R_{k} U_{1, k}, \\
R_{2, k}= & \mathcal{E}\left[v_{2, k} v_{2, k}^{T}\right]=U_{2, k}^{T} R_{k} U_{2, k}, \\
R_{12}(k, k)= & \mathcal{E}\left[v_{1, k} v_{2, k}^{T}\right]=0, \\
R_{12}(k, i)= & \mathcal{E}\left[v_{1, k} v_{2, i}^{T}\right]=0 \text { for } \quad k \neq i .
\end{aligned}
$$

Moreover, Cheng et al. (2009) show the following relations:

- $\operatorname{cov}\left[v_{1, k}, w_{i}\right]=0$ and $\operatorname{cov}\left[v_{2, k}, w_{i}\right]=0$ for $k \neq i$.

- $\operatorname{cov}\left[v_{1, k}, x_{0}\right]=0$ and $\operatorname{cov}\left[v_{2, k}, x_{0}\right]=0$.

Under the system equations (4)-(6), we note that we can estimate the second component of the fault not at the step $k$ but at the step $k-1$, because $f_{2, k-1}$ will be estimated from the state. The proposed state and fault filter has the following structure:

$$
\begin{aligned}
\hat{x}_{k / k-1}= & A_{k-1} \hat{x}_{k-1 / k-1}+B_{k-1} u_{k-1} \\
& +F_{1, k-1}^{x} \hat{f}_{1, k-1}, \\
\hat{f}_{2, k-1}= & K_{k}^{f_{2}}\left(z_{2, k}-H_{2, k} \hat{x}_{k / k-1}\right), \\
\hat{x}_{k / k-1}^{*}= & \hat{x}_{k / k-1}+F_{2, k-1}^{x} \hat{f}_{2, k-1} \\
& +K_{k}^{x *}\left(z_{2, k}-H_{2, k} \hat{x}_{k / k-1}\right), \\
\hat{f}_{1, k}= & K_{k}^{f_{1}}\left(z_{1, k}-H_{1, k} \hat{x}_{k / k-1}^{*}\right), \\
\hat{f}_{k}= & V_{1, k} \hat{f}_{1, k}+V_{2, k} \hat{f}_{2, k-1}, \\
\hat{x}_{k / k}= & \hat{x}_{k / k-1}^{*}+K_{k}^{x}\left(z_{2, k}-H_{2, k} \hat{x}_{k / k-1}^{*}\right),
\end{aligned}
$$

where the gain matrices $K_{k}^{f_{1}} \in \mathbb{R}^{r_{k} \times r_{k}}, K_{k}^{f_{2}} \in$ $\mathbb{R}^{\left(p-r_{k}\right) \times\left(m-r_{k}\right)}, \quad K_{k}^{x *} \in \mathbb{R}^{n \times\left(m-r_{k}\right)}$ and $K_{k}^{x} \in$ $\mathbb{R}^{n \times\left(m-r_{k}\right)}$ still have to be determined to satisfy the following criteria:

- Unbiasedness: The estimator must satisfy

$$
\begin{aligned}
\mathcal{E}\left[\tilde{f}_{k}\right] & =\mathcal{E}\left[f_{k}-\hat{f}_{k}\right]=0, \\
\mathcal{E}\left[\tilde{x}_{k / k}\right] & =\mathcal{E}\left[x_{k}-\hat{x}_{k / k}\right]=0 .
\end{aligned}
$$

- Minimum-variance: The estimator is determined such that

- the mean square errors $\mathcal{E}\left[\left(\tilde{f}_{k}\right)^{T} \tilde{f}_{k}\right]$ is minimized subject to the constraint (16),

- $\operatorname{trace}\left\{P_{k / k}^{x}=\mathcal{E}\left[\tilde{x}_{k / k}\left(\tilde{x}_{k / k}\right)^{T}\right]\right\}$ is minimized subject to the constraints (16) and (17). 


\section{Filter design}

In this section, the gain matrices $K_{k}^{f_{1}}, K_{k}^{f_{2}}, K_{k}^{x}$ and $K_{k}^{x *}$ will be determined so that the filter (10)-(15) yields robust estimates of $f_{k}$ and $x_{k}$ in spite of the presence of the unknown disturbances $d_{k}$. Next, the UMV fault and state estimation will be demonstrated.

3.1. Fault estimation. The estimation errors of $f_{1, k}$ and $f_{2, k-1}$ are given by

$$
\begin{aligned}
\tilde{f}_{1, k}= & f_{1, k}-\hat{f}_{1, k} \\
= & \left(I_{r_{k}}-K_{k}^{f_{1}} \Sigma_{k}\right) f_{1, k}-K_{k}^{f_{1}} e_{1, k}, \\
\tilde{f}_{2, k-1}= & f_{2, k-1}-\hat{f}_{2, k-1} \\
= & \left(I_{p-r_{k}}-K_{k}^{f_{2}} H_{2, k} F_{2, k-1}^{x}\right) f_{2, k-1} \\
& -K_{k}^{f_{2}} H_{2, k} E_{k-1}^{x} d_{k-1}-K_{k}^{f_{2}} e_{2, k},
\end{aligned}
$$

where

$$
\begin{aligned}
e_{1, k}= & H_{1, k} \tilde{x}_{k / k-1}^{*}+v_{1, k}, \\
\tilde{x}_{k / k-1}^{*}= & x_{k}-\hat{x}_{k / k-1}^{*}, \\
e_{2, k}= & H_{2, k} \tilde{\bar{x}}_{k / k-1}+v_{2, k}, \\
\tilde{\bar{x}}_{k / k-1}= & A_{k-1} \tilde{x}_{k-1 / k-1} \\
& +F_{1, k-1}^{x} \tilde{f}_{1, k-1}+w_{k-1}, \\
\tilde{x}_{k / k}= & x_{k}-\hat{x}_{k / k} .
\end{aligned}
$$

Referring to (18) and (19), the estimator $\hat{f}_{k}$ given by (14) is unbiased if and only if $K_{k}^{f_{1}}$ and $K_{k}^{f_{2}}$ satisfy the following constraints:

$$
\begin{aligned}
& K_{k}^{f_{1} \Sigma_{k}}=I_{r_{k}}, \\
& K_{k}^{f_{2}} G_{k}=\mathcal{F}_{k},
\end{aligned}
$$

where

$$
G_{k}=\left[H_{2, k} F_{2, k-1}^{x} \quad H_{2, k} E_{k-1}^{x}\right]
$$

and

$$
\mathcal{F}_{k}=\left[\begin{array}{ll}
I_{p-r_{k}} & 0_{\left(p-r_{k}\right) \times q}
\end{array}\right] .
$$

Let $\tilde{x}_{k / k-1}^{*}, \tilde{x}_{k-1 / k-1}$ and $\tilde{f}_{1, k-1}$ be unbiased. The covariance matrices of $e_{1, k}$ and $e_{2, k}$ are defined respectively by

$$
\begin{aligned}
\tilde{R}_{1, k} & =\mathcal{E}\left[e_{1, k} e_{1, k}^{T}\right] \\
& =H_{1, k} P_{k / k-1}^{x^{*}} H_{1, k}^{T}+R_{1, k}, \\
\tilde{R}_{2, k} & =\mathcal{E}\left[e_{2, k} e_{2, k}^{T}\right] \\
& =H_{2, k} \bar{P}_{k / k-1}^{x} H_{2, k}^{T}+R_{2, k},
\end{aligned}
$$

where

$$
P_{k / k-1}^{x^{*}}=\mathcal{E}\left[\tilde{x}_{k / k-1}^{*} \tilde{x}_{k / k-1}^{* T}\right]
$$

and

$$
\bar{P}_{k / k-1}^{x}=\mathcal{E}\left[\tilde{\bar{x}}_{k / k-1} \tilde{\bar{x}}_{k / k-1}^{T}\right]
$$

Since neither $e_{1, k}$, nor $e_{2, k}$ has unit variances, the Least-Squares (LS) solutions do not have a minimumvariance. For that, $f_{1, k}$ and $f_{2, k}$ can be obtained by weighted least-squares estimation (Kailath et al., 2000; Gillijns and Moor, 2007b) with two weighting matrices $\tilde{R}_{1, k}^{-1}$ and $\tilde{R}_{2, k}^{-1}$. Then, to have unbiased fault estimates, the matrices gain $K_{k}^{f_{1}}$ and $K_{k}^{f_{2}}$ are obtained as follows:

$$
\begin{aligned}
K_{k}^{f_{1}} & =\left(\Sigma_{k}^{T} \tilde{R}_{1, k}^{-1} \Sigma_{k}\right)^{-1} \Sigma_{k}^{T} \tilde{R}_{1, k}^{-1}, \\
K_{k}^{f_{2}} & =\mathcal{F}_{k} G_{k}^{*},
\end{aligned}
$$

where

$$
G_{k}^{*}=\left(G_{k}^{T} \tilde{R}_{2, k}^{-1} G_{k}\right)^{+} G_{k}^{T} \tilde{R}_{2, k}^{-1}
$$

is the generalized inverse of the matrix $G_{k}$.

The variances of the WLS solutions (18) and (19) are respectively given by

$$
\begin{aligned}
& P_{k}^{f_{1}}=\mathcal{E}\left[\tilde{f}_{1, k}\left(\tilde{f}_{1, k}\right)^{T}\right]=\left(\Sigma_{k}^{T} \tilde{R}_{1, k}^{-1} \Sigma_{k}\right)^{-1}, \\
& P_{k}^{f_{2}}=\mathcal{E}\left[\tilde{f}_{2, k-1}\left(\tilde{f}_{2, k-1}\right)^{T}\right]=K_{k}^{f_{2}} \tilde{R}_{2, k}\left(K_{k}^{f_{2}}\right)^{T},
\end{aligned}
$$

Referring to Eqns. (8), (14), (18) and (19), the fault error estimate $\tilde{f}_{k}$ has the following form:

$$
\tilde{f}_{k}=\left[\begin{array}{ll}
V_{1, k} & V_{2, k}
\end{array}\right]\left[\begin{array}{c}
\tilde{f}_{1, k} \\
\tilde{f}_{2, k}
\end{array}\right] .
$$

Using (32), the covariance matrix $P_{k}^{f}$ is given by

$$
P_{k}^{f}=\left[\begin{array}{ll}
V_{1, k} & V_{2, k}
\end{array}\right]\left[\begin{array}{cc}
P_{k}^{f_{1}} & P_{k}^{f_{12}} \\
P_{k}^{f_{21}} & P_{k}^{f_{2}}
\end{array}\right]\left[\begin{array}{c}
V_{1, k}^{T} \\
V_{2, k}^{T}
\end{array}\right],
$$

where

$$
\begin{aligned}
P_{k}^{f_{12}} & =\left(P_{k}^{f_{21}}\right)^{T}=\mathcal{E}\left[\tilde{f}_{1, k} \tilde{f}_{2, k-1}^{T}\right] \\
& =K_{k}^{f_{1}} H_{1, k}\left[\bar{P}_{k / k-1}^{x} H_{2, k}^{T}+\tilde{S}_{k}\right]\left(K_{k}^{f_{2}}\right)^{T}
\end{aligned}
$$

with

$$
\tilde{S}_{k}=\mathcal{E}\left[\tilde{x}_{k / k-1}^{*} v_{2, k}^{T}\right]=-\left(F_{k-1}^{x} K_{k}^{f_{2}}+K_{k}^{x *}\right) \tilde{R}_{2, k} .
$$

3.2. State estimation. Referring to Eqns. (1) and (12), the state estimation error $\tilde{x}_{k / k-1}^{*}$ is defined as

$$
\begin{aligned}
\tilde{x}_{k / k-1}^{*}= & \tilde{\bar{x}}_{k / k-1}-\left(K_{k}^{x *}+F_{2, k-1}^{x} K_{k}^{f_{2}}\right) e_{2, k} \\
& +\left(E_{k-1}^{x}-K_{k}^{x *} H_{2, k} E_{k-1}^{x}\right) d_{k-1} \\
& -K_{k}^{x *} H_{2, k} F_{2, k-1}^{x} f_{2, k-1} .
\end{aligned}
$$

The estimator $\hat{x}_{k / k-1}^{*}(12)$ is unbiased if $K_{k}^{x *}$ satisfies the following constraint to eliminate the terms $f_{2, k-1}$ and $d_{k-1}$ from the error estimate (35):

$$
K_{k}^{x *} G_{k}=\mathcal{F}_{k}^{*}
$$


where

$$
G_{k}=\left[H_{2, k} F_{2, k-1}^{x} \quad H_{2, k} E_{k-1}^{x}\right]
$$

and

$$
\mathcal{F}_{k}^{*}=\left[0_{n \times\left(p-r_{k}\right)} \quad E_{k-1}^{x}\right]
$$

Lemma 1. A necessary and sufficient condition for the estimators (4) and (5) to be unbiased is that matrix $G_{k}$ has full column rank, i.e.,

$$
\operatorname{rank}\left(G_{k}\right)=\operatorname{rank}\left(F_{2, k-1}^{x}\right)+\operatorname{rank}\left(E_{k-1}^{x}\right) .
$$

Proof. Equations (24) and (36) can be written as

$$
\left[\begin{array}{l}
K_{k}^{f_{2}} \\
K_{k}^{x *}
\end{array}\right] G_{k}=\left[\begin{array}{l}
\mathcal{F}_{k} \\
\mathcal{F}_{k}^{*}
\end{array}\right] .
$$

A necessary and sufficient condition for the existence of the solution to (38) is

$$
\operatorname{rank}\left[\begin{array}{c}
\mathcal{F}_{k} \\
\mathcal{F}_{k}^{*} \\
G_{k}
\end{array}\right]=\operatorname{rank}\left(G_{k}\right)
$$

We expand (39) and obtain

$$
\begin{aligned}
& \operatorname{rank}\left[\begin{array}{c}
\mathcal{F}_{k} \\
\mathcal{F}_{k}^{*} \\
G_{k}
\end{array}\right]=\operatorname{rank}\left[\begin{array}{cc}
I_{p-r_{k}} & 0_{\left(p-r_{k}\right) \times q} \\
0_{n \times\left(p-r_{k}\right)} & E_{k-1}^{x} \\
H_{2, k} F_{2, k-1}^{x} & H_{2, k} E_{k-1}^{x}
\end{array}\right] \\
& =\operatorname{rank}\left[H_{2, k} F_{2, k-1}^{x} \quad H_{2, k} E_{k-1}^{x}\right] \\
& =\operatorname{rank}\left(H_{2, k} F_{2, k-1}^{x}\right)+\operatorname{rank},\left(H_{2, k} E_{k-1}^{x}\right)
\end{aligned}
$$

Finally, referring to the assumption (7), we will have

$$
\operatorname{rank}\left(G_{k}\right)=\operatorname{rank}\left(F_{2, k-1}^{x}\right)+\operatorname{rank}\left(E_{k-1}^{x}\right) .
$$

However, this can be easily justified by considering that the faults and the unknown disturbances have independent influences.

Referring to (35) and (36), the covariance matrix $P_{k / k-1}^{x *}$ has the following form:

$$
\begin{aligned}
P_{k / k-1}^{x *} & \\
= & \mathcal{E}\left[\tilde{x}_{k / k-1}^{*}\left(\tilde{x}_{k / k-1}^{*}\right)^{T}\right] \\
= & \left(I_{n}-F_{2, k-1}^{x} K_{k}^{f_{2}} H_{2, k}-K_{k}^{x *} H_{2, k}\right) \bar{P}_{k / k-1}^{x} \\
& \times\left(I_{n}-F_{2, k-1}^{x} K_{k}^{f_{2}} H_{2, k}-K_{k}^{x *} H_{2, k}\right)^{T} \\
& +\left(F_{2, k-1}^{x} K_{k}^{f_{2}}+K_{k}^{x *}\right) R_{2, k}\left(F_{2, k-1}^{x} K_{k}^{f_{2}}+K_{k}^{x *}\right)^{T} .
\end{aligned}
$$

The gain matrix $K_{k}^{x *}$ is determined by minimizing the trace of the covariance matrix $P_{k / k-1}^{x *}$ (37) such that (36) is satisfied. Using the Kitanidis method (Kitanidis, 1987), we obtain

$$
\begin{aligned}
& {\left[\begin{array}{cc}
\tilde{R}_{2, k} & -G_{k} \\
G_{k}^{T} & 0
\end{array}\right]\left[\begin{array}{c}
\left(K_{k}^{x *}\right)^{T} \\
\left(\Lambda_{k}^{*}\right)^{T}
\end{array}\right]} \\
& \quad=\left[\begin{array}{c}
-\tilde{R}_{2, k}\left(K_{k}^{f_{2}}\right)^{T}\left(F_{2, k-1}^{x}\right)^{T}+H_{2, k} \bar{P}_{k / k-1}^{x} \\
\mathcal{F}_{k}^{*}
\end{array}\right],
\end{aligned}
$$

where $\Lambda_{k}^{*}$ is the matrix of Lagrange multipliers.

Equation (38) will have a unique solution. Accordingly, the gain matrix $K_{k}^{x *}$ is given by

$$
\begin{aligned}
K_{k}^{x *}= & {\left[\bar{P}_{k / k-1}^{x} H_{2, k}^{T}-F_{2, k-1}^{x} K_{k}^{f_{2}} \tilde{R}_{2, k}\right] } \\
& \times \tilde{R}_{2, k}^{-1}\left(I-G_{k} G_{k}^{*}\right)+\mathcal{F}_{k}^{*} G_{k}^{*},
\end{aligned}
$$

where $G_{k}^{*}$ was defined in (29).

Using (1) and (15), the state estimation error $\tilde{x}_{k / k}$ has the following form:

$$
\begin{aligned}
\tilde{x}_{k / k} & =x_{k}-\hat{x}_{k / k} \\
& =\left(I-K_{k}^{x} H_{2, k}\right) \tilde{x}_{k / k-1}^{*}-K_{k}^{x} v_{2, k} .
\end{aligned}
$$

Considering (40), the covariance matrix $P_{k / k}^{x}$ is determined as follows:

$$
\begin{aligned}
P_{k / k}^{x} & =\mathcal{E}\left[\tilde{x}_{k / k} \tilde{x}_{k / k}^{T}\right] \\
& =P_{k / k-1}^{x *}+K_{k}^{x} R_{k}^{*} K_{k}^{x T}-V_{k}^{*} K_{k}^{x T}-K_{k}^{x} V_{k}^{* T},
\end{aligned}
$$

where

$$
\begin{aligned}
R_{k}^{*}= & H_{2, k} P_{k / k-1}^{x *} H_{2, k}^{T}+R_{2, k}+H_{2, k} S_{k}^{*} \\
& +\left(H_{2, k} S_{k}^{*}\right)^{T}, \\
V_{k}^{*}= & P_{k / k-1}^{x *}\left(H_{2, k}\right)^{T}+S_{k}^{*}, \\
S_{k}^{*}= & \mathcal{E}\left[\tilde{x}_{k / k-1}^{*} v_{2, k}^{T}\right] \\
= & -\left(F_{2, k-1}^{x} K_{k}^{f 2}+K_{k}^{x *}\right) R_{2, k} .
\end{aligned}
$$

In order to obtain a minimum-variance estimate, we have to minimize the trace of (41). Thus, the gain matrix $K_{k}^{x}$ is given by

$$
K_{k}^{x}=\left(P_{k / k-1}^{x *} H_{2, k}^{T}+S_{k}^{*}\right) \alpha_{k}^{T}\left(\alpha_{k} R_{k}^{*} \alpha_{k}^{T}\right)^{-1} \alpha_{k} .
$$

where $\alpha_{k}$ is an arbitrary matrix which has to be chosen such that $\alpha_{k} R_{k}^{*} \alpha_{k}^{T}$ has full rank (Gillijns and Moor, 2007a; Darouach et al., 2003).

3.3. Filter time update. From Eqn. (22), the covariance matrix $\bar{P}_{k / k-1}^{x}$ has the following form:

$$
\begin{aligned}
\bar{P}_{k+1 / k}^{x} & =\mathcal{E}\left[\tilde{\bar{x}}_{k+1 / k}\left(\tilde{\bar{x}}_{k+1 / k}\right)^{T}\right] \\
& =\left[\begin{array}{ll}
A_{k} & F_{1, k}^{x}
\end{array}\right]\left[\begin{array}{cc}
P_{k / k}^{x} & P_{k}^{x f_{1}} \\
\left(P_{k}^{x f_{1}}\right)^{T} & P_{k}^{f_{1}}
\end{array}\right]\left[\begin{array}{c}
A_{k}^{T} \\
F_{1, k}^{x T}
\end{array}\right]+Q_{k},
\end{aligned}
$$

where $P_{k}^{x f_{1}}$ is calculated by using (18) and (40). Then, we obtain

$$
\begin{aligned}
P_{k}^{x f_{1}} & =\mathcal{E}\left[\tilde{x}_{k / k}\left(\tilde{f}_{1, k}\right)^{T}\right] \\
& \left.=\left(K_{k}^{x}\left(V_{k}^{*}\right)^{T}-P_{k / k-1}^{x *}\right)\left(K_{k}^{f 1} H_{1, k}\right)\right)^{T} .
\end{aligned}
$$




\section{Applications}

In this section, we propose the use of the resulting filter to solve the problem of estimating simultaneous actuator and sensor faults and to make a comparison with the existing literature results, in particular the ones of Ben Hmida et al. (2010).

4.1. Robust estimation of simultaneous actuator and sensor faults. We consider the numerical example used by Chen and Patton (1996; 1999). The linearized model of a simplified longitudinal flight control system is the following:

$$
\begin{aligned}
x_{k+1}= & \left(A_{k}+\Delta A_{k}\right) x_{k}+\left(B_{k}+\Delta B_{k}\right) u_{k} \\
& +F_{k}^{a} f_{k}^{a}+w_{k}, \\
y_{k}= & H_{k} x_{k}+F_{k}^{s} f_{k}^{s}+v_{k},
\end{aligned}
$$

where the state variables are the pitch angle $\delta_{z}$, the pitch rate $\omega_{z}$ and the normal velocity $\eta_{y}$. The control input $u_{k}$ is the elevator control signal. $F_{k}^{a}$ and $F_{k}^{s}$ denote the matrices defining the distribution of the actuator fault $f_{k}^{a}$ and the sensor fault $f_{k}^{s}$, respectively. The presented system equations can be rewritten as follows:

$$
\begin{aligned}
x_{k+1} & =A_{k} x_{k}+B_{k} u_{k}+F_{k}^{x} f_{k}+E_{k}^{x} d_{k}+w_{k}, \\
y_{k} & =H_{k} x_{k}+F_{k}^{y} f_{k}+v_{k},
\end{aligned}
$$

where $F_{k}^{x}$ and $F_{k}^{y}$ represent the matrices defining the injection of the faults vector in the state and measurement equations:

$$
F_{k}^{x}=\left[\begin{array}{ll}
F_{k}^{a} & 0
\end{array}\right], \quad F_{k}^{y}=\left[\begin{array}{ll}
0 & F_{k}^{s}
\end{array}\right] .
$$

The term $E_{k}^{x} d_{k}$ represents the parameter perturbation in matrices the $A_{k}$ and $B_{k}$ :

$$
E_{k}^{x} d_{k}=\Delta A_{k} x_{k}+\Delta B_{k} u_{k}
$$

The system parameter matrices are

$$
\begin{aligned}
& A_{k}=\left[\begin{array}{ccc}
0.9944 & -0.1203 & -0.4302 \\
0.0017 & 0.9902 & -0.0747 \\
0 & 0.8187 & 0
\end{array}\right] \\
& B_{k}=\left[\begin{array}{c}
0.4252 \\
-0.0082 \\
0.1813
\end{array}\right] \text {, } \\
& H_{k}=I_{3 \times 3}, x_{k}=\left[\begin{array}{lll}
\eta_{y} & \omega_{z} & \delta_{z}
\end{array}\right]^{T}, \\
& Q_{k}=\operatorname{diag}\left\{0.1^{2}, 0.1^{2}, 0.01^{2}\right\}, \quad R_{k}=0.1^{2} I_{3 \times 3}
\end{aligned}
$$

We inject simultaneously two faults in the system,

$$
\left[\begin{array}{l}
f_{k}^{a} \\
f_{k}^{s}
\end{array}\right]=\left[\begin{array}{c}
4 u_{s}(k-20)-4 u_{s}(k-60) \\
-2 u_{s}(k-30)+2 u_{s}(k-70)
\end{array}\right],
$$

where $u_{s}(k)$ is the unit-step function. The first fault, $f_{k}^{a}$, occurs in the actuator between time instants 20 and 60, and the second fault, $f_{k}^{s}$, occurs in the sensor for $\delta_{z}$ between time instants 30 and 70 . Thus, we consider the cases of single and multiples faults. The unknown disturbance is given by

$$
E_{k}^{x} d_{k}=E_{k}^{x}\left\{\left[\begin{array}{lll}
\Delta a_{21} & \Delta a_{22} & \Delta a_{23}
\end{array}\right] x_{k}+\Delta b_{2} u_{k}\right\}
$$

where $\Delta a_{i j}$ and $\Delta b_{i}(i=1$ and $j=1,2,3)$ are perturbations in aerodynamic and control coefficients. The matrices defining injection of the fault and the unknown disturbances are

$$
E_{k}^{x}=\left[\begin{array}{l}
0 \\
1 \\
0
\end{array}\right], \quad F_{k}^{a}=\left[\begin{array}{c}
0.4252 \\
-0.0082 \\
0.1813
\end{array}\right], \quad F_{k}^{s}\left[\begin{array}{l}
0 \\
0 \\
1
\end{array}\right]
$$

In the simulation, the aerodynamic coefficients are perturbed by $\pm 50 \%$, i.e., $\Delta a_{i j}=-0.5 a_{i j}$ and $\Delta b_{i}=0.5 b_{i}$. In addition, we set $u_{k}=10, x_{0}=0, P_{0}=0.1^{2} I_{3 \times 3}$.

In Fig. 1, the actual and estimated values of the state vector $x_{k}=\left[\eta_{y} \omega_{z} \delta_{z}\right]^{T}$ are displayed. Figure 2 presents the actual and estimated values of the first element and the second element of the fault vector $f_{k}=\left[\begin{array}{ll}f_{k}^{a} & f_{k}^{s}\end{array}\right]^{T}$, respectively. In Table 1 the Root Mean Square Errors
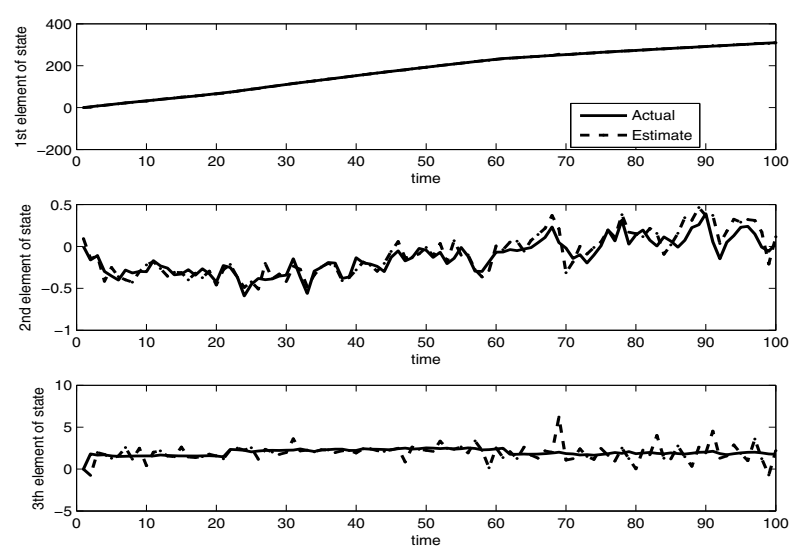

Fig. 1. Actual state $x_{k}$ and the estimated state $\hat{x}_{k}$.

(RMSEs) of the states $x_{k}=\left[\eta_{y} \omega_{z} \delta_{z}\right]^{T}$ and the faults $f_{k}=\left[\begin{array}{ll}f_{k}^{a} & f_{k}^{s}\end{array}\right]^{T}$ are given along with the traces of their steady-input and state estimation error covariances. Ac-

Table 1. Performance of the proposed filter.

\begin{tabular}{|c|c|c|c|c|}
\hline$x^{1}$ & $x^{2}$ & $x^{3}$ & $f^{a}$ & $f^{s}$ \\
\hline \hline 0.7467 & 0.1059 & 0.8966 & 1.1198 & 0.2159 \\
\hline
\end{tabular}

cording to the simulation results (Figs. 1 and 2 and Table 1), it can be seen that the proposed filter produces better estimates of the state and faults. We primarily focus on simultaneous estimation of actuator and sensor faults in spite of the presence of unknown disturbances. 

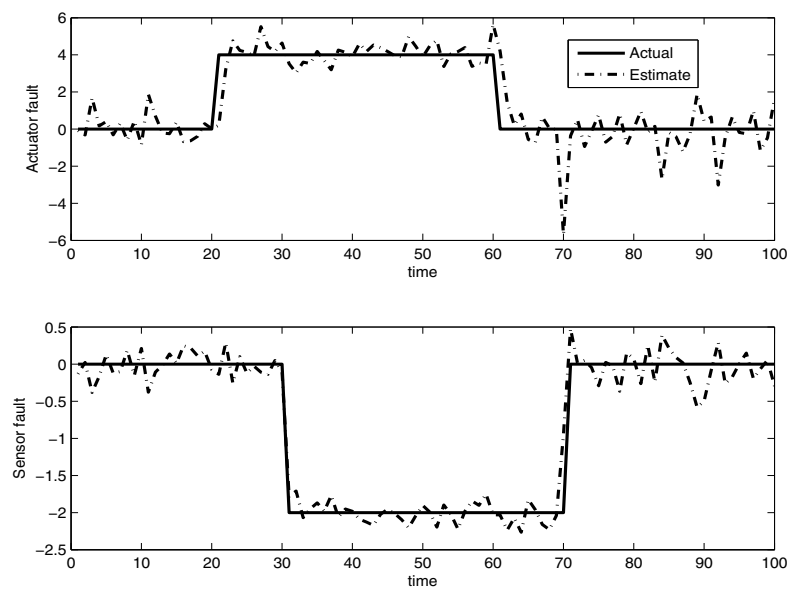

Fig. 2. Actual fault $f_{k}$ and the estimated fault $\hat{f}_{k}$.

4.2. Comparative study. We consider the numerical example used by Ben Hmida et al. (2010). In this simulation, three cases of $F_{k}^{y}$ will be studied:

$$
\begin{gathered}
\left(F_{k}^{y}\right)^{1}=\left[\begin{array}{cc}
2 & 1 \\
0.6 & 0.3 \\
0.2 & 0.1
\end{array}\right], \quad\left(F_{k}^{y}\right)^{2}=\left[\begin{array}{cc}
2 & 0 \\
0.6 & 0 \\
0.2 & 0
\end{array}\right], \\
\left(F_{k}^{y}\right)^{3}=\left[\begin{array}{ll}
0 & 1.4 \\
0 & 0.3 \\
0 & 1.6
\end{array}\right]
\end{gathered}
$$

dealing with particular ranks of the matrix $F_{k}^{y}$. given by

We assume that the fault and the disturbance are

$$
\begin{aligned}
{\left[\begin{array}{l}
f_{k}^{1} \\
f_{k}^{2}
\end{array}\right] } & =\left[\begin{array}{l}
4 u_{s}(k-25)-4 u_{s}(k-70) \\
5 u_{s}(k-30)-5 u_{s}(k-65)
\end{array}\right], \\
d_{k} & =4 u_{s}(k-15)-4 u_{s}(k-55),
\end{aligned}
$$

where $u_{s}(k)$ is the unit-step function.

Figures 3 and 4 present the actual and estimated values of the first and second elements of the fault vector $f_{k}=\left[\begin{array}{ll}f_{k}^{1} & f_{k}^{2}\end{array}\right]^{T}$, respectively. In Tables 2$]$ and 3 , the RMSE value of the states $x_{k}=\left[x_{k}^{1} x_{k}^{2} x_{k}^{3}\right]$ and the fault $f_{k}=\left[f_{k}^{1} f_{k}^{2}\right]^{T}$ are given along with the traces of their steady-fault and state estimation error covariance matrices. According to the simulation results in Figs. 3 and 4 and Tables 2 and 3 , we may conclude with the following results:

1. In all cases, the EUMV filter (Ben Hmida et al., 2010) and the proposed filter give the same values of the RMSE of the state estimation error. On the other hand, the proposed filter gives smaller RMSE values of the fault estimation errors.
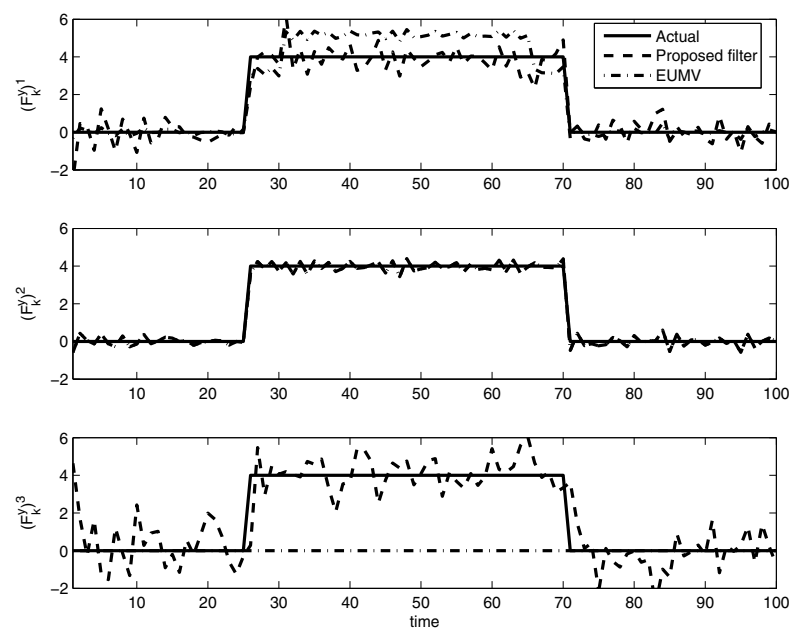

Fig. 3. Actual fault $f_{k}^{1}$ and the estimated fault $\hat{f}_{k}^{1}$.
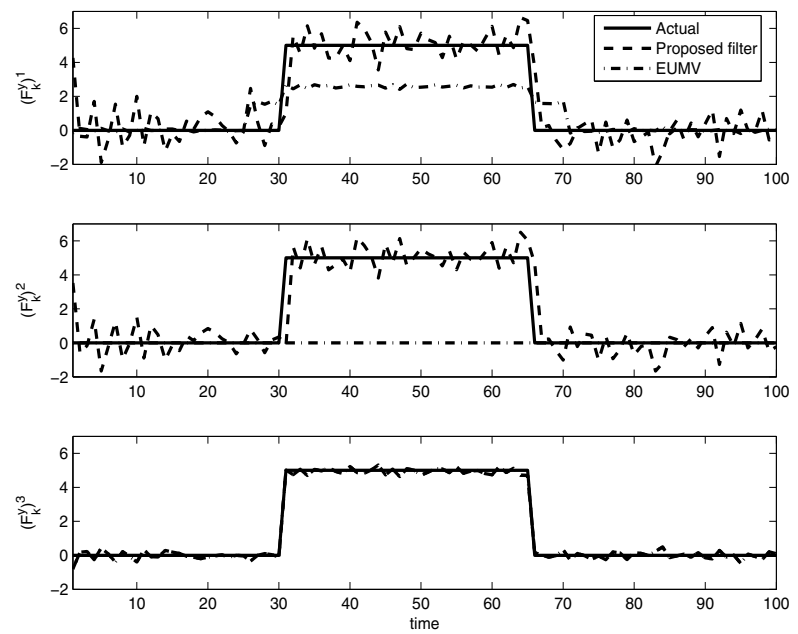

Fig. 4. Actual fault $f_{k}^{2}$ and the estimated fault $\hat{f}_{k}^{2}$.

2. When referring to the traces of the steady-fault estimation error covariance, it appears that

$$
\begin{aligned}
\operatorname{trace}\left(P^{f}(\mathrm{EUMV} \text { filter })\right) \\
\quad=\operatorname{trace}\left(P^{f_{1}}(\text { Proposed filter })\right) \\
\leq \operatorname{trace}\left(P^{f}(\text { Proposed filter })\right) .
\end{aligned}
$$

Clearly, the fault estimation obtained by the EUMV filter is in fact only the first component estimate $\hat{f}_{1, k}$ in our proposed filter. Dealing with the other component of the fault $f_{2, k}$, our proposed filter improves the overall performance, despite a superior trace of the steady-fault estimation error covariance as, logically, 
Table 2. Evaluation of the RMSE values.

\begin{tabular}{|c|l|c|c|c|c|c|}
\hline$F_{k}^{y}$ & Filter & $x_{k}^{1}$ & $x_{k}^{2}$ & $x_{k}^{3}$ & $f_{k}^{1}$ & $f_{k}^{2}$ \\
\hline \hline \multirow{2}{*}{$\left(F_{k}^{y}\right)^{1}$} & EUMV filter & 0.5248 & 0.1346 & 0.1863 & 0.7577 & 1.5179 \\
& Proposed filter & 0.5248 & 0.1346 & 0.1863 & 0.6588 & 1.0942 \\
$\left(F_{k}^{y}\right)^{2}$ & EUMV filter & 0.6301 & 0.1542 & 0.2129 & 0.2565 & 2.9580 \\
& Proposed filter & 0.6301 & 0.1542 & 0.2129 & 0.2565 & 1.0434 \\
$\left(F_{k}^{y}\right)^{3}$ & EUMV filter & 0.3378 & 0.0938 & 0.3647 & 2.6833 & 0.2103 \\
& Proposed filter & 0.3378 & 0.0938 & 0.3647 & 1.1992 & 0.2103 \\
\hline
\end{tabular}

Table 3. Trace of steady fault and state estimation error covariances.

\begin{tabular}{|c|l|c|c|c|c|}
\hline$F_{k}^{y}$ & Filter & $\operatorname{trace}\left(P_{k / k}^{x}\right)$ & $\operatorname{trace}\left(P_{k / k}^{f 1}\right)$ & $\operatorname{trace}\left(P_{k / k}^{f 2}\right)$ & $\operatorname{trace}\left(P_{k / k}^{f}\right)$ \\
\hline \hline \multirow{2}{*}{$\left(F_{k}^{y}\right)^{1}$} & EUMV filter & 0.2645 & - & - & 0.0301 \\
& Proposed filter & 0.2645 & 0.0301 & 0.8068 & 0.8370 \\
$\left(F_{k}^{y}\right)^{2}$ & EUMV filter & 0.3732 & - & - & 0.0525 \\
& Proposed filter & 0.3732 & 0.0525 & 0.4361 & 0.4885 \\
$\left(F_{k}^{y}\right)^{3}$ & EUMV filter & 0.1977 & - & - & 0.0338 \\
& Proposed filter & 0.1977 & 0.0338 & 0.8350 & 0.8688 \\
\hline
\end{tabular}

$$
\begin{aligned}
& \operatorname{trace}\left(P^{f}(\text { Proposed filter })\right) \\
& =\operatorname{trace}\left(P^{f}(\text { EUMV filter })\right) \\
& \quad+\operatorname{trace}\left(P^{f 2}(\text { Proposed filter })\right) .
\end{aligned}
$$

\section{Conclusion}

In this paper, the problem of joint state and fault estimation for linear time-varying stochastic systems with unknown disturbances was solved. To achieve this objective, a new recursive optimal unbiased minimum-variance filter was proposed when the direct feedthrough matrix of the fault has arbitrary rank. The advantages of this filter are especially important in the case when we do not have any prior information about the fault and the unknown disturbances. This filter is applied efficiently to solve two problems. Firstly, it estimates the actuator and sensor faults simultaneously. Secondly, it establishes a comparative study with the existing literature results.

\section{References}

Ben Hmida, F., Khémiri, K., Ragot, J. and Gossa, M. (2010). Unbiased minimum-variance filter for state and fault estimation of linear time-varying systems with unknown disturbances, Mathematical Problems in Engineering, Vol. 2010, Article ID 343586, 17 pages.

Blanke, M., Kinnaert, M., Lunze, J. and Staroswiecki, M. (2006). Diagnosis and Fault-Tolerant Control, SpringerVerlag, Berlin/Heidelberg.

Chen, J. and Patton, R. (1996). Optimal filtering and robust fault diagnosis of stochastic system with unknown disturbances, IEE Proceedings: Control Theory Application 143(1): 31-36.
Chen, J. and Patton, R. (1999). Robust Model-based Fault Diagnosis for Dynamic Systems, Kluwer Academic Publishers, Norwell, MA.

Cheng, Y., Ye, H., Wang, Y. and Zhou, D. (2009). Unbiased minimum-variance state estimation for linear systems with unknown input, Automatica 45(2): 485-491.

Darouach, M. and Zasadzinski, M. (1997). Unbiased minimum variance estimation for system with unknown exogenous inputs, Automatica 33(4): 717-719.

Darouach, M., Zasadzinski, M. and Boutayeb, M. (2003). Extension of minimum variance estimation for systems with unknown inputs, Automatica 39(5): 867-876.

Gillijns, S. and Moor, B. (2007a). Unbiased minimum-variance input and state estimation for linear discrete-time systems with direct feedthrough, Automatica 43(5): 934-937.

Gillijns, S. and Moor, B. (2007b). Unbiased minimum-variance input and state estimation for linear discrete-time systems, Automatica 43(1): 111-116.

Hou, M. and Patton, R.J. (1998). Optimal filtering for system with unknown inputs, IEEE Transactions on Automatic Control 43(3): 445-449.

Hsieh, C. (2009). Extension of unbiased minimum-variance input and state estimation for systems with unknown inputs, Automatica 45(9): 2149-2153.

Hsieh, C.S. (2000). Robust two-stage Kalman filters for systems with unknown inputs, IEEE Transactions on Automatic Control 45(12): 2374-2378.

Jamouli, H., Keller, J. and Sauter, D. (2003). Fault isolation filter with unknown inputs in stochastic systems, Proceedings of Safeprocess, Washington, DC, USA, pp. 531-536.

Kailath, T., Sayed, A. and Hassibi, B. (2000). Linear Estimation, Prentice Hall, Englewood Cliffs, NJ.

Keller, J. (1998). Fault isolation filter design for linear stochastic systems with unknown inputs, 37th IEEE Conference on Decision and Control, Tampa, FL, USA, pp. 598-603. 
Keller, J. (1999). Fault isolation filter design for linear stochastic systems, Automatica 35(10): 1701-1706.

Keller, J. and Sauter, D. (2011). Restricted diagonal detection filter and updating strategy for multiple fault detection and isolation, International Journal of Adaptive Control and Signal Processing 25(1): 68-87.

Kitanidis, P. (1987). Unbiased minimum-variance linear state estimation, Automatica 23(6): 775-778.

Nikoukhah, R. (1994). Innovation generation in the presence of unknown inputs: Application to robust failure detection, Automatica 30(12): 1851-1867.

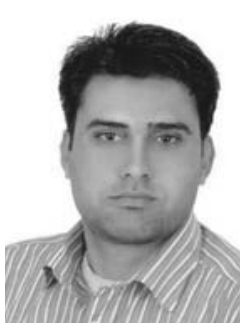

Karim Khémiri was born in 1982 in Jendouba, Tunisia. He received a B.Sc. in electrical engineering from the High School of Sciences and Techniques of Tunis (ESSTT) at Tunis University in 2005. In 2007, he obtained the Master's degree in automatic control at the same university. Currently, he is working toward a Ph.D. in the research unit on control, monitoring and safety of systems at ESSTT. His main research interests are in Kalman filtering, and fault detection and diagnosis.

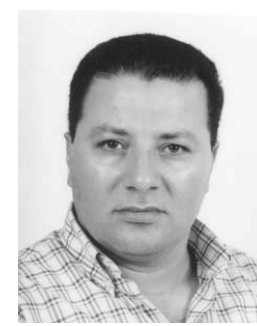

Fayçal Ben Hmida was born in 1966 in Sfax, Tunisia. He received a B.Sc. in electrical engineering from the High Normal School of Technical Education of Tunis (ENSET) in 1991. In 1992, he obtained the Master's degree in automatics and informatics at Aix-Marseille III University, France, and a Ph.D. in engineering production and informatics at the same university in 1997. In 1998, he joined the High School of Sciences and Techniques of Tunis (ESSTT) at Tunis University as an assistant professor. Now, he is a member of the Research Unit on Control, Monitoring and Safety of Systems (C3S) at ESSTT. His main research interests are fault detection and isolation, robust estimation and robust filtering.

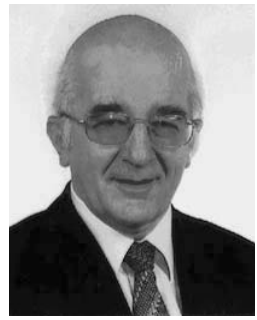

José Ragot is a researcher in the Research Center for Automatic Control of Nancy. His major research fields include data validation and reconciliation, process diagnosis, fault detection and isolation. A part of his activities is devoted to modeling and state estimation, adapted to process diagnosis and mainly in the field of multi-models. He has successfully advised $63 \mathrm{Ph} . \mathrm{D}$. and $3 \mathrm{HDR}$ (French D.Sc.) theses, and has published about 500 refereed technical communications including 94 papers in international journals, 280 communications in international conferences, 4 books and 12 chapters in collective volumes. Applications have been in various fields such as mineral and metallurgical processing, chemical engineering, water treatment, aerospace, environmental processes.

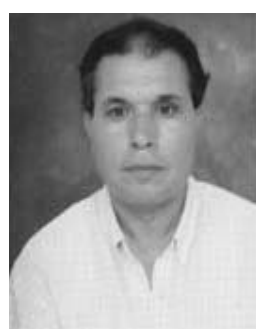

Moncef Gossa was born in Kairouan, Tunisia, in 1957. He received the B.Sc. and DEA degrees from the High Normal School of Technical Education of Tunis (ENSET), in 1981 and 1982 respectively, the Ph.D. degree from the INSA of Toulouse, France, in 1984, and the Habilitation à Diriger des Recherches (HDR) degree from the National Engineering School of Tunis (ENIT), in 2004, all in electrical engineering. Currently, he is a professor at ESSTT, the director of the Higher Institute of Technological Studies (ISET) of Radès and the director of the Research Unit on Control, Monitoring and Safety of Systems (C3S). He has authored more than 60 papers published in international conference proceedings and technical journals in the area as well as many patents. His research is in the fields of electrical machines, sensorless vector control of AC motor drives and diagnosis.

Received: 6 December 2010 Revised: 6 July 2011 